\title{
Clinical evidence of abscopal effect in cutaneous squamous cell carcinoma treated with diffusing alpha emitters radiation therapy: a case report
}

\author{
Salvatore Roberto Bellia, MD!, Giacomo Feliciani², Massimo Del Duca, MD', Manuela Monti ${ }^{3}$, Valentina Turri, MD4, \\ Anna Sarnelli, PhD², Antonino Romeo, MD!, Prof. Itzhak Kelson5', Prof. Yona Keisarib, Prof. Aron Popovtzer?, \\ Toni Ibrahim, MD, PhD8, Prof. Giovanni Paganelli?, Prof. Ignazio Stanganellili
}

'Department of Radiotherapy, Istituto Scientifico Romagnolo per lo Studio e la Cura dei Tumori (IRST) IRCCS, Meldola (FC), Italy. ${ }^{2}$ Department of Medical Physics, Istituto Scientifico Romagnolo per lo Studio e la Cura dei Tumori (IRST) IRCCS, Meldola (FC), Italy, ${ }^{3}$ Biostatistics and Clinical Experimentation, Istituto Scientifico Romagnolo per lo Studio e la Cura dei Tumori (IRST) IRCCS, Meldola (FC), Italy, ${ }^{4}$ Medical Direction, Istituto Scientifico Romagnolo per lo Studio e la Cura dei Tumori (IRST) IRCCS, Meldola (FC), Italy, ${ }^{5}$ School of Physics and Astronomy, Tel Aviv University, Exact Sciences, Tel Aviv, Israel, 'Sackler Faculty of Medicine, Tel Aviv University, Clinical Microbiology and Immunology, Tel Aviv, Israel, ${ }^{7}$ Department of Radiation Oncology, David of Cancer Center and Sackler Faculty of Medicine, Israel University, Tel Aviv, Israel, ${ }^{8}$ Osteoncology and Rare Tumors Center CDO-TR, Istituto Scientifico Romagnolo per lo Studio e la Cura dei Tumori (IRST) IRCCS, Meldola (FC), Italy, ${ }^{9}$ Nuclear Medicine Unit, Istituto Scientifico Romagnolo per lo Studio e la Cura dei Tumori (IRST) IRCCS, Meldola (FC), Italy, ${ }^{10}$ Skin Cancer Unit, Istituto Scientifico Romagnolo per lo Studio e la Cura dei Tumori (IRST) IRCCS, Meldola (FC), Italy

\begin{abstract}
Purpose: Alpha particle treatments could enhance the probability of an immune response, which can lead to abscopal effects (AE). We report a case of a patient affected by multiple cutaneous squamous cell carcinoma (cSCC). After the treatment with diffusing alpha emitters radiation therapy (DaRT) of one lesion, an AE was observed on at least two distant ones.

Material and methods: We investigated a case of a 65-year-old female patient with multiple synchronous lesions of the skin of lower limbs confirmed by a biopsy. Patient was enrolled in a clinical trial N.CTP-SCC-00 (NCT03015883), with the objective to assess effectiveness of DaRT technique. DaRT is based on the insertion of locally ${ }^{224}$ Ra-loaded seeds in a clinical target volume (CTV). Treatment plan with positron emission tomography/computed tomography (PET/CT) was used to entirely cover the CTV. Follow-up and biopsy evaluations were employed to outline the patient outcome.

Results: We performed seeds implantation according to the Paris system. At $28^{\text {th }}$ day, an evident lesion shrinkage with a persistent minimal area of hyperkeratosis was noted. 76 days after implantation, a complete remission of the treated lesion was observed and an evident reduction of the area with two more distant lesion, which could be associated to an immune-mediated response. One year after the treatment, a complete remission of treated lesion was observed as well as spontaneous regression of untreated distant ones.

Conclusions: In this study, we reported evidences of an AE in cSCC stimulated by radiation and possibly mediated by immune system. In the next DaRT treatments, our intent is to monitor T-lymphocytes variations in peripheral blood in order to demonstrate indirect activation of the immune system mediated by radiation also in patients with solitary lesions, in which, by definition, an AE cannot be observed.

Key words: alpha particles, abscopal effect, cutaneous squamous cell carcinoma, brachytherapy.

\section{Purpose}

The abscopal effect (AE) is a sporadic event of tumor regression following radiotherapy treatment observed at distance from irradiated site. For the first time, the term 'abscopal effect' was used by Mole in 1953 [1]. The word
'abscopal' comes from Latin 'ab' and 'scopos', which
means 'position away from the target'. This effect is a rare
clinical event, however, there have been reports of this
effect in various type of cancers, not only in solid tumors

Received: 31.05 .2019

Address for correspondence: Giacomo Feliciani, Department of Medical Physics, Istituto Scientifico $\begin{array}{ll}\text { Romagnolo per lo Studio e la Cura dei Tumori (IRST) IRCCS, Via Piero Maroncelli 40, 47014, Meldola (FC), Accepted: } 25.07 .2019 \\ \text { Italy, phone: +39 3274730398, } ₫ \text { e-mail: giacomo.feliciani@irst.emr.it } & \text { Published: } 30.10 .2019\end{array}$
Published: 30.10 .2019 
but also in leukemias and lymphomas [2,3,4]. In particular, for skin tumors, there have been reports of a case of Merkel cell carcinoma and several cases of melanoma $[5,6,7,8,9,10]$. Recently, there has been growing evidence that high linear energy transfer (LET) radiations, such as alpha particles, are more prone to leverage this effect rather than low-LET X-rays usually used in external beam radiation therapy (EBRT) [11,12]. We report a case of a patient affected by cutaneous squamous cell carcinoma (cSCC). This patient had multiple synchronous lesions and after the treatment of one of the lesion with diffusing alpha emitters radiotherapy (DaRT), an abscopal effect was observed on two distant lesions. DaRT used in interstitial brachytherapy is an effective and short treatment [13]. As skin cancer incidence is rising worldwide and the interest in brachytherapy applications is growing continuously $[14,15]$, we decided to propose this treatment as an alternative to surgery. Patient refused surgery, since it was already applied multiple times [16]. Furthermore, we didn't consider external beam radiotherapy; in our opinion, expected toxicity and duration of the treatment would have been higher [17].

\section{Material and methods}

\section{Patient description}

We present a case of a Caucasian 65-year-old female patient who presented with multiple synchronous epithelial lesions of the lower limbs skin. She lived for three years (between the $24^{\text {th }}$ and $26^{\text {th }}$ year of age) in the Caribbean and most probably was overexposed to ultraviolet (UV) radiations. From the age of 58, she developed many metachronous epithelial cutaneous lesions that were surgically excised. Most of them were CSCC and a small percentage were keratoacanthomas (KA) confirmed by a biopsy. She did not experience any significant comorbidity. The patient denied spontaneous resolution of any individual lesion. Clinical suspect of multiple cSCC was confirmed by anatomo-pathological equipe on the most relevant lesions A and B (Figure 1). Lesion A in Figure 1 is characterized by a keratinizing erythematous nodule of about $15 \mathrm{~mm}$, whereas lesion B shows mostly the same characteristics but it's larger $(18 \mathrm{~mm})$ and exophytic. Lesion A was qualified as G1, and lesion B as G2.

As a treatment, we proposed the enrollment in the international 'first in man' clinical trial N.CTP-SCC-00 (NCT03015883), which has the objective of assessing the safety and effectiveness of DaRT technique [18]. This approach involves standard interstitial brachytherapy technique, with high relative biological effectiveness (RBE) of alpha particles.

\section{Pretreatment examination}

The lesions were digitally photographed and physically measured with a ruler prior to DaRT seeds insertion. Imaging was done on a SIEMENS Biograph MCT 20 flow 4R PET/CT scanner using a standard PET/CT clinical protocol. Patient has fasted for at least 6 hours before performing intravenous administration of 18F-FDG (3.7 MBq/ kg). Serum glucose concentrations were measured before FDG injection and were less than $200 \mathrm{mg} / \mathrm{dl}$. After PET/CT scan imagining, gross tumor volume (GTV) contouring was performed by a radiotherapist. CTV is generated from GTV with an isotropic expansion of $5 \mathrm{~mm}$ to account for microscopic subclinical disease for this histology type. Physical and radiological measurements are taken as a reference for baseline lesion extension. In further treatments with different kind of histology and tumor volumes, different margin can be considered according to the radiotherapy recommendations for non-melanoma skin cancer [19].

\section{DaRT description}

DaRT is an interstitial brachytherapy technique in treatments of solid tumors with the insertion of $1 \mathrm{~cm}$ long ${ }^{224} \mathrm{Ra}$-loaded seeds, as shown in Figure 2. ${ }^{224} \mathrm{Ra}$ decays into ${ }^{220} \mathrm{Rn}$, which has the ability to diffuse into the tumor, with an average activity of $100 \mathrm{kBq}$ each at calibration [20]. These $1 \mathrm{~cm}$ long seeds are attached to sutures
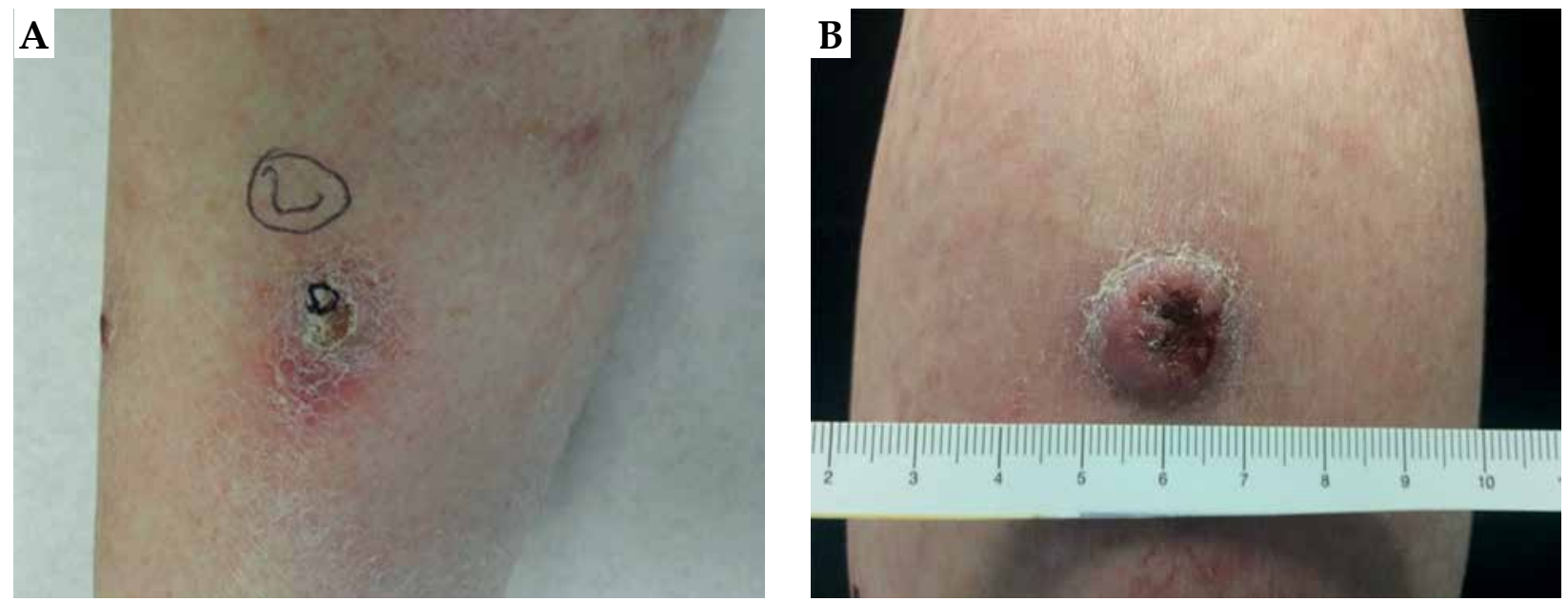

Fig. 1. Lesion A (A) characterized by a keratinizing erythematous nodule of about $15 \mathrm{~mm}$ and lesion $\mathrm{B}(\mathbf{B})$ that is slightly larger $(18 \mathrm{~mm})$, with the same characteristics and exophytic 

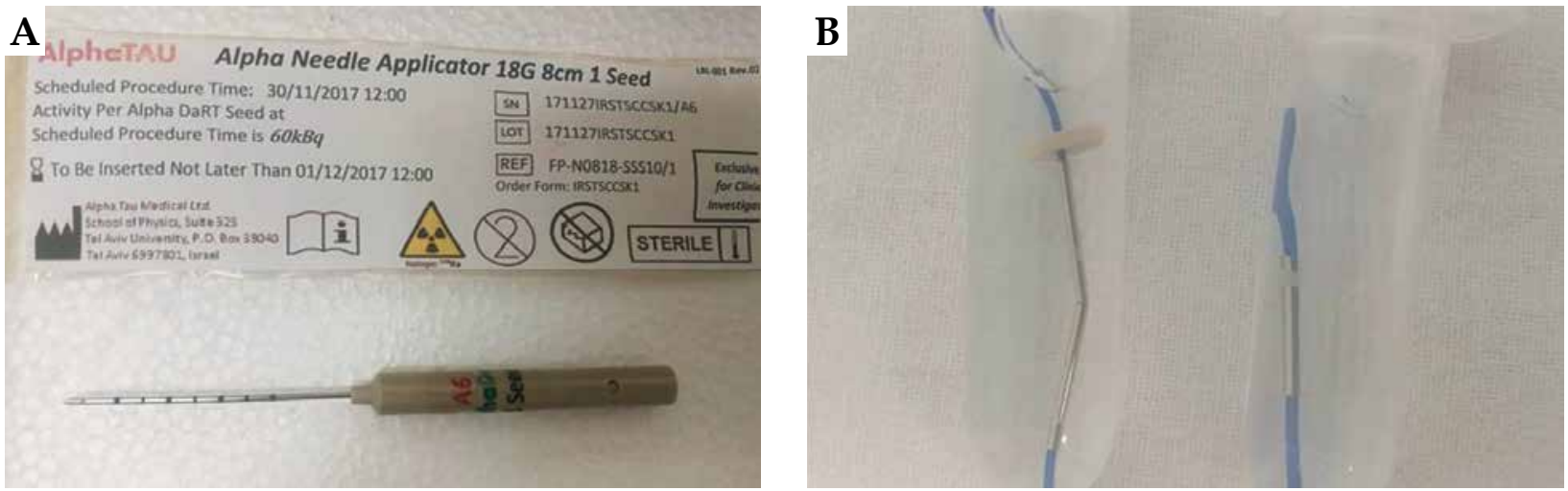

Fig. 2. Standard alpha needle applicator (A) with its sealed case showing on the label all the details about length of the needle, number of seeds, seed activity, and serial number. B - The appearance of iron seeds loaded with radium-224 and blue wires for suture

that are inserted directly in the tumor site by means of interstitial needles (Figure 2). ${ }^{220} \mathrm{Rn}$ is released by recoil from ${ }^{216} \mathrm{Po}$ and ${ }^{212} \mathrm{~Pb}$ atoms. ${ }^{220} \mathrm{Rn}$ migrate to a radial distance of about 2-2.5 $\mathrm{mm}$ from the seed in tumor diffusing daughter-atoms and alpha particles, leading to the formation of an high absorbed dose region of at least $10 \mathrm{~Gy}$ in 15 days after implant, not considering the relative biological effectiveness (RBE) of alpha particles [20,21,22]. After this period, the implant is removed.

Few alpha particles passing by the cell nucleus are sufficient to destroy its membrane leading to cell death; their RBE is actually considered much higher and thus released dose is increased. Furthermore, their effectiveness is not dependent neither on oxygenation nor on cell cycle phase of the tumor cells.

High destructive power of alpha radiation comes from double string DNA damage and indirectly from vasculature rupture. Recurrence of treated tumor may depend on angiogenesis. The inhibition of major pro-angiogenic factors and multiple angiogenesis associated processes may be increased by high-RBE particles [11,23]. Due to these features, alpha particles are mainly effective against CSCs (cancer stem cells) that are considered more resistant to low-RBE ionizing radiation of EBRT [24].

\section{Treatment planning procedure}

On the basis of PET/CT images, a treatment plan was created to evaluate appropriate quantity of seeds to entirely cover the CTV during the intervention. Oncentra brachytherapy treatment planning system was used in order to correctly arrange the required number of seeds, and to estimate the possible entrance points of needles. Figure 3A shows the treatment planning procedure. The seeds, each loaded with $100 \mathrm{kBq}$ of ${ }^{224} \mathrm{Ra}$, were placed according to geometrical arrangement of the Paris system that was adapted to the variable size of DaRT seeds, ranging from $1 \mathrm{~cm}$ to $6 \mathrm{~cm}$ in size and comparing to linear sources. The interspacing is modified according to the Paris system, with an optimal interspacing of $14 \mathrm{~mm}$ to $5 \mathrm{~mm}$ due to the alpha emission nature of ${ }^{224} \mathrm{Ra}$ source. Precise dose calculation cannot be performed as a commercial TPS for alpha particles, since dose deposi- tion evaluation is not available; however, as stated in [20], a coverage of $10 \mathrm{~Gy}$ is guaranteed at a radial distance of $2.5 \mathrm{~mm}$. Furthermore, as per our experience, it is useful to perform a geometrical arrangement evaluation before the interventional treatment, with correct evaluation of the depth of lesion. If the lesion depth is above $5 \mathrm{~mm}$, it is necessary to deliver the treatment with two or more different planes. Therefore, we estimated 5 needle applicators, loaded with 2 seeds for lesion A (15 mm diameter and $4.5 \mathrm{~mm}$ in depth, 10 seeds in total), and 5 needle applicators loaded with 2 seeds and 4 needle applicators loaded with 1 seed for lesion B (18 mm diameter and $6.5 \mathrm{~mm}$ in depth, 14 seeds in total).

\section{Results}

\section{Interventional treatment description}

Our initial intention was to treat during the same session the most relevant lesions A and B. However, in the day of implantation, lesion A appeared to be clearly enlarged (about $2 \times 3 \mathrm{~cm}$ ) and we decided to treat only lesion A to ensure sufficient amount of seeds to adequately cover the target. Due to lesion enlargement, we had a mismatch between our treatment plan and the actual lesion dimension. Therefore, we planned to subsequently treat lesion $\mathrm{B}$, with close monitoring during frequent follow-up visits as required by the protocol. Prior local anesthesia with $20 \mathrm{ml}$ mepivacain + adrenalin $1: 100,000$ along the perimeter of the lesion, we contoured the CTV directly on the skin, and we performed the seeds implantation according to the Paris system: a total of 8 applicators (7 loaded with 2 seeds and 1 with 1 seed, for a total number of 15 seeds) were inserted with an intra-seed wire distance of about $5 \mathrm{~mm}$ on a single plane, 7 parallels, and 1 perpendicular to adequately cover the peripheral margin of CTV, as shown in Figure 4. The mismatch between the treatment planning and the actual treatment resulted in 5 seeds. After the implant, we performed a CT scan to verify the adequate geometrical disposition of the seeds inside the tumor, as preplanned. In Figures 3C and 3D, the difference between planning PET/CT and delivered treatment after lesion growth is presented. We would 

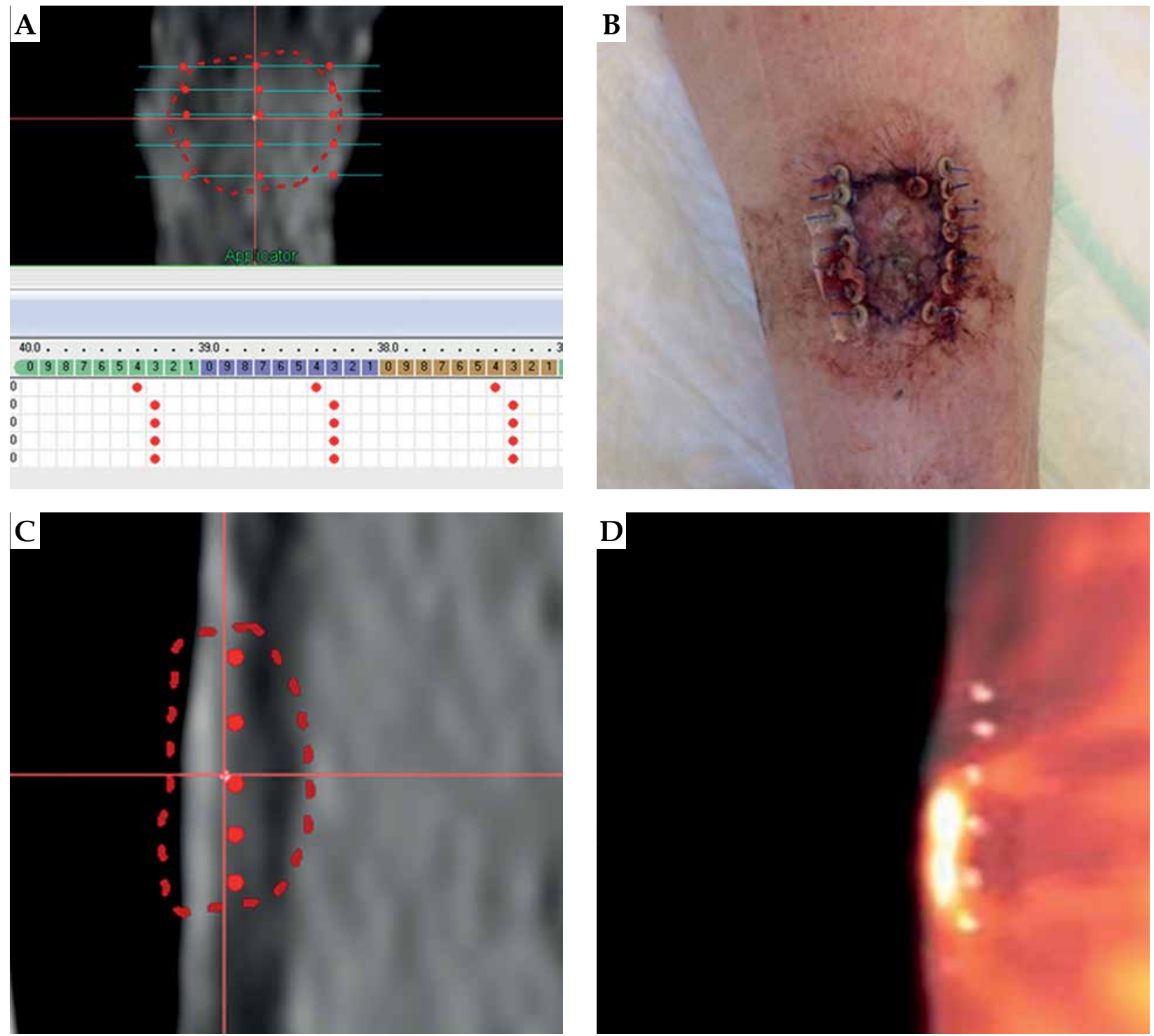

Fig. 3. A) The treated lesion B that has grown from treatment planning session; B) The pretreatment planning session, 5 needles of 2 seeds spaced between each other of about 3-4 mm to account for lesion swelling were foreseen for the CTV. The implant resulted in 7 needles with 2 seeds, 1 needle with 1 seed, for a total of 15 seeds. This underlines the importance of having spare needles before starting a procedure; C) Saggital view of the treatment planning; D) The fusion between preplanning PET and after treatment CT. An evident lesion growth between PET scan and intervention day

like to emphasize the importance of this early verification through CT scan because in case of unsatisfactory results, it is possible to 'correct the shot' and alter the position of any seed, which is in a different location than expected, or inserting any other/s for a better coverage of the entire CTV. However, the best imaging to evaluate tumor extension is to employ high frequency US during the implant procedure. After 15 days, $95 \%$ of the radiation was delivered and the seeds were removed. Generally, the removal is performed at day 15 , and to deliver $100 \%$ of dose, 15 more days would be required, possibly resulting in uncomfortable tolerance level of the patient and unnecessary to reach the tumor killing dose.

Figure 5 (left side) shows an evident lesion shrinkage with a persistent minimal area of hyperkeratosis. To compare, the untreated lesion B in Figure 6.
Fifteen days after the treatment of lesion A, lesion B showed a noticeable reduction of its nodular component persisted mild hyperkeratosis with perilesional erythema. On the $28^{\text {th }}$ day, a dermatologist performed a re-biopsy of untreated lesion $\mathrm{B}$ and the residual lesion $\mathrm{A}$, in accordance with suspected minimal area of persistence of disease, as shown in Figure 7. On the same date, a biopsy of a third lesion (C) was performed, on the contra-lateral thigh shown in Figure 7, in the perspective of a possible new DaRT treatment. Only lesion C resulted cSCC positive.

Histological examination of both, the treated lesion A and the untreated lesion $B$, showed no residual malignant cells. Following the complete tumor remission of the treated lesion $\mathrm{A}$ and the spontaneous regression of lesion $\mathrm{B}$, we decided to plan a treatment for the third lesion $\mathrm{C}$. 

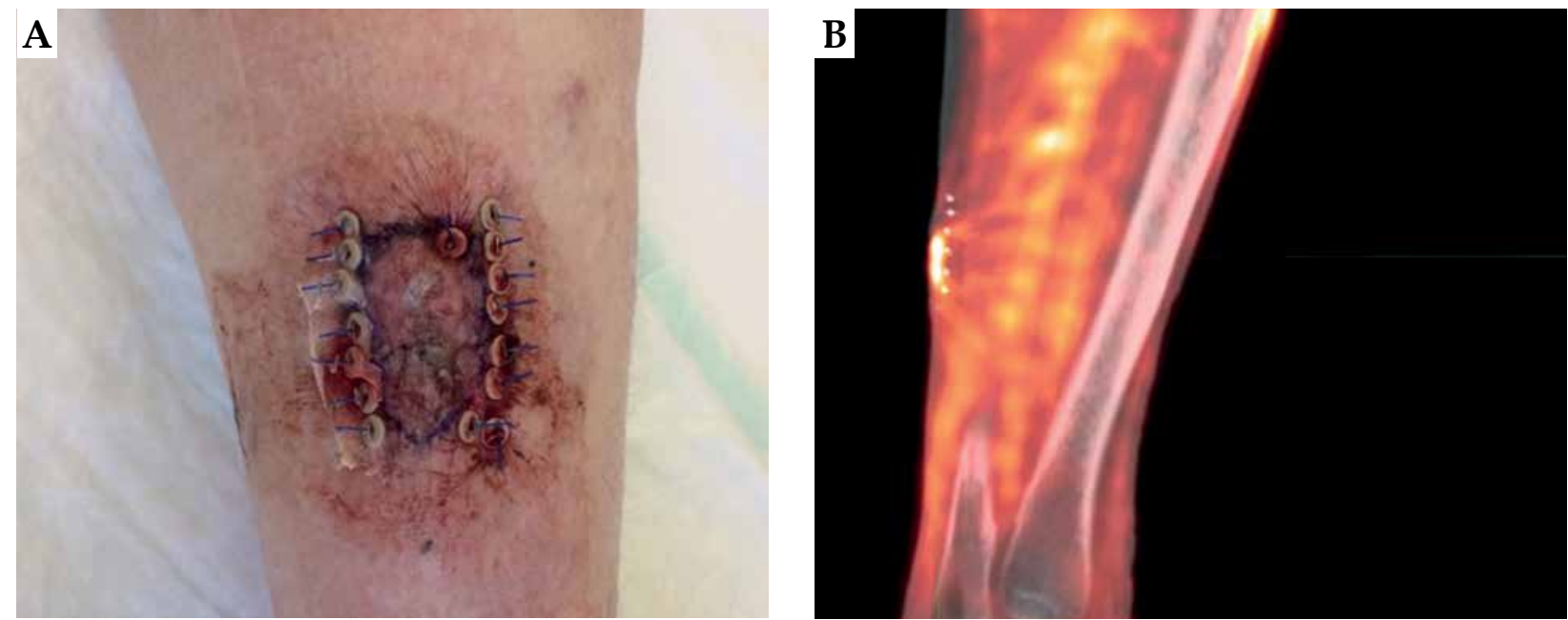

Fig. 4. A) Final appearance of lesion A one day after treatment; B) Fusion between the pretreatment PET/CT and the CT performed after the implant
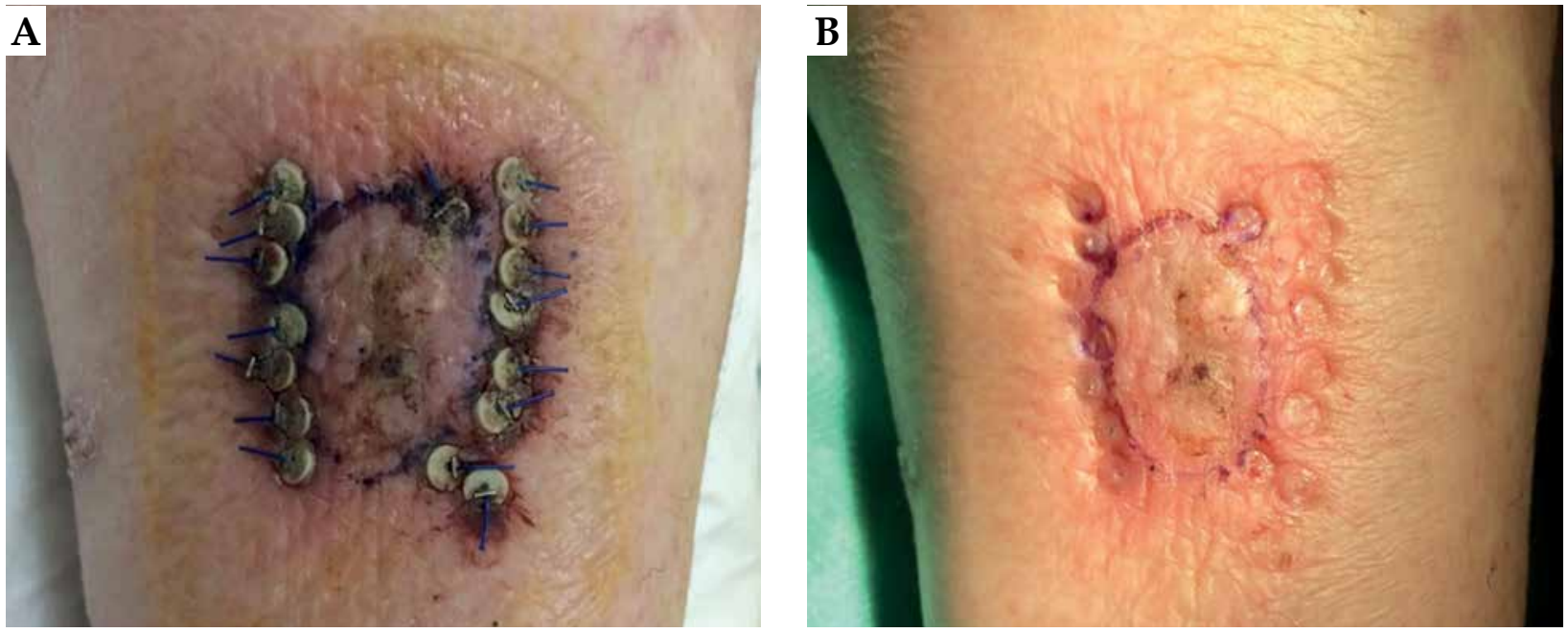

Fig. 5. Appearance of lesion A at day 15 before (A) and immediately after (B) implant removal

However, two and a half months after the implant and about 10 days before the planned DaRT treatment, in a joint evaluation with dermatologist, we noted that lesion $\mathrm{C}$ was also in regression (as shown in Figure 8 right) and the scheduled treatment was canceled. Figure 8 shows the left side of the appearance of lesion A, B in the center, and $C$ on the right, 76 days after the treatment. While the patient achieved a complete response in the targeted irradiated lesion (A left), an out-of-target tumor response in at least two distant sites (B and $\mathrm{C}$ ) was noted. Lesion B was located about $15 \mathrm{~cm}$ from lesion A, whereas lesion $C$ was located on the contralateral leg. One year after the treatment, a complete remission of treated lesion persists as well as spontaneous regression of untreated distant lesions.

Clinically, patient reported only a grade 1 erythema and a mild degree transitory pain in implant location during the 15 days of implant. No other adverse event occurred after implant removal.

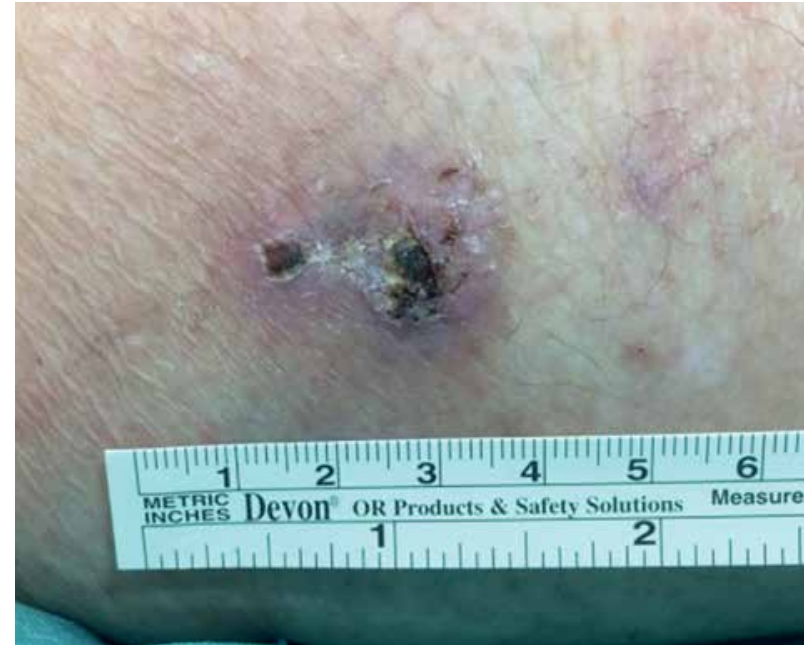

Fig. 6. Modification of lesion B at $15^{\text {th }}$ day after implant: marked reduction of its nodular component, with persisting mild hyperkeratosis with perilesional erythema 

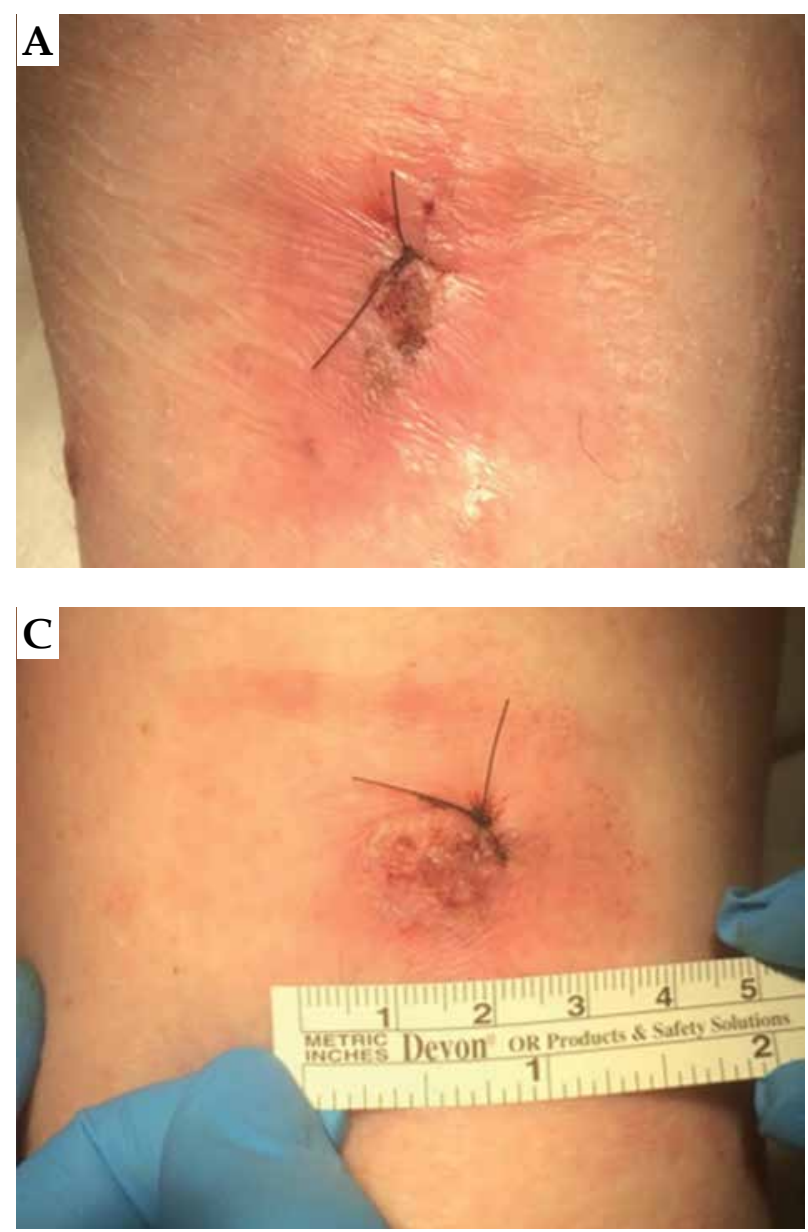

\section{Discussion}

The mechanism of AE possibly associated with enhanced immune effect triggered by high-dose radiation, has not been clearly defined and is still not well understood. There are many theories on the abscopal effect. The first concept refers to lymphomas and leukemias; it is hypothesized that irradiation may lead to a release of substances toxic to lymphoma, and inhibit the production and secretion of substances responsible for continuation of tumor growth $[25,26,27]$. The second theory applies to solid tumors, where local irradiation induces a release of cytokines (mitotic inhibitors), such as tumor necrosis factor (TNF), into the circulation that mediate a systemic antitumor effect $[1,28]$. The third suggests that the abscopal effect is immune-mediated: irradiation of tumor in one site induces local release of circulating tumor antigens (CTA) and/or inflammatory factors that may mediate an increased immune response against unirradiated lesion that express similar tumor antigens; furthermore, local radiotherapy would augment the activity of natural killer cells [29]. The last and most suggestive hypothesis, also named 'in vivo vaccination', is that local tumor damage expose hidden tumoral antigens that are able to induce systemic antitumor immunity [29,30,31]. Accumulating evidences suggest that a dysfunction in the host systemic immunity and/or low immunogenic characteristics of the

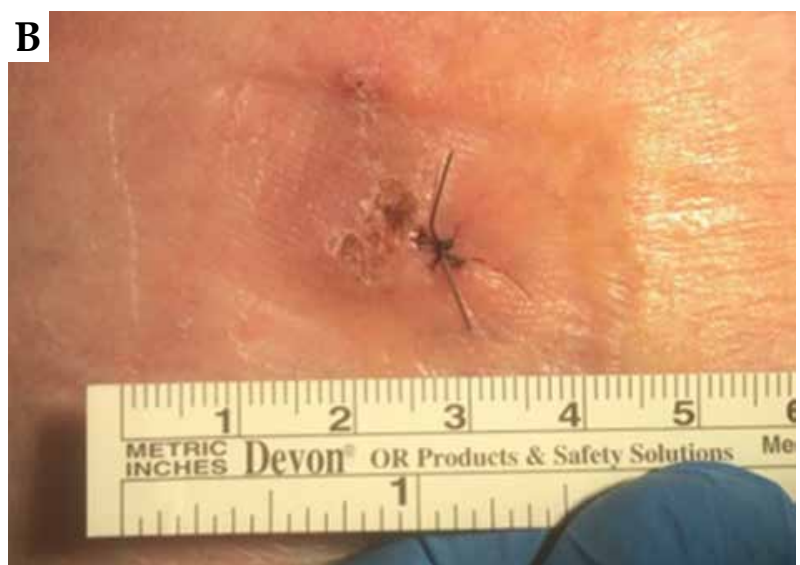

Fig. 7. A) Re-biopsy on residual lesion A, in correspondence of suspected minimal area of persistence of disease; B) Re-biopsy of untreated lesion B; C) Biopsy of a third lesion, on the contra-lateral thigh, for a possible new DaRT treatment

tumor may favor the progression of cancer. Anti-tumor immune-mediated mechanisms are operative during tumor development and immune surveillance play an important role in cancer control. Tumor-derived immunosuppression constantly diminishes anti-tumor immune response.

Therefore, a treatment capable of restoring or strengthening the immune functions might constitute an effective antineoplastic treatment. Recent studies reports that radiotherapy can act as an immunostimulant, causing (for example) the immunogenic cell death and releasing the danger signals such as HMGB1 [32,33]. Thanks to the intrinsic characteristics of high-LET alpha particles, with its ability to induce an aggressive in situ tumor destruction (potent tumor ablation), DaRT treatment may play a very important role because it could stimulate an anti-tumor immune reactivity with more ease than low-LET radiation that is used with conventional external beam radiation therapy (EBRT). Furthermore, the destruction of the tumor by DaRT maintains an intact vasculature around the tumor, enabling an influx of immune cells to recognize and destroy tumor cells.

If confirmed, this ability could open up new scenarios for DaRT, also in a clinical situation, in which radiation treatment is usually considered of very little use. For instance, in cases where tumoricidal doses on whole tumoral mass with standard irradiation techniques is not pos- 

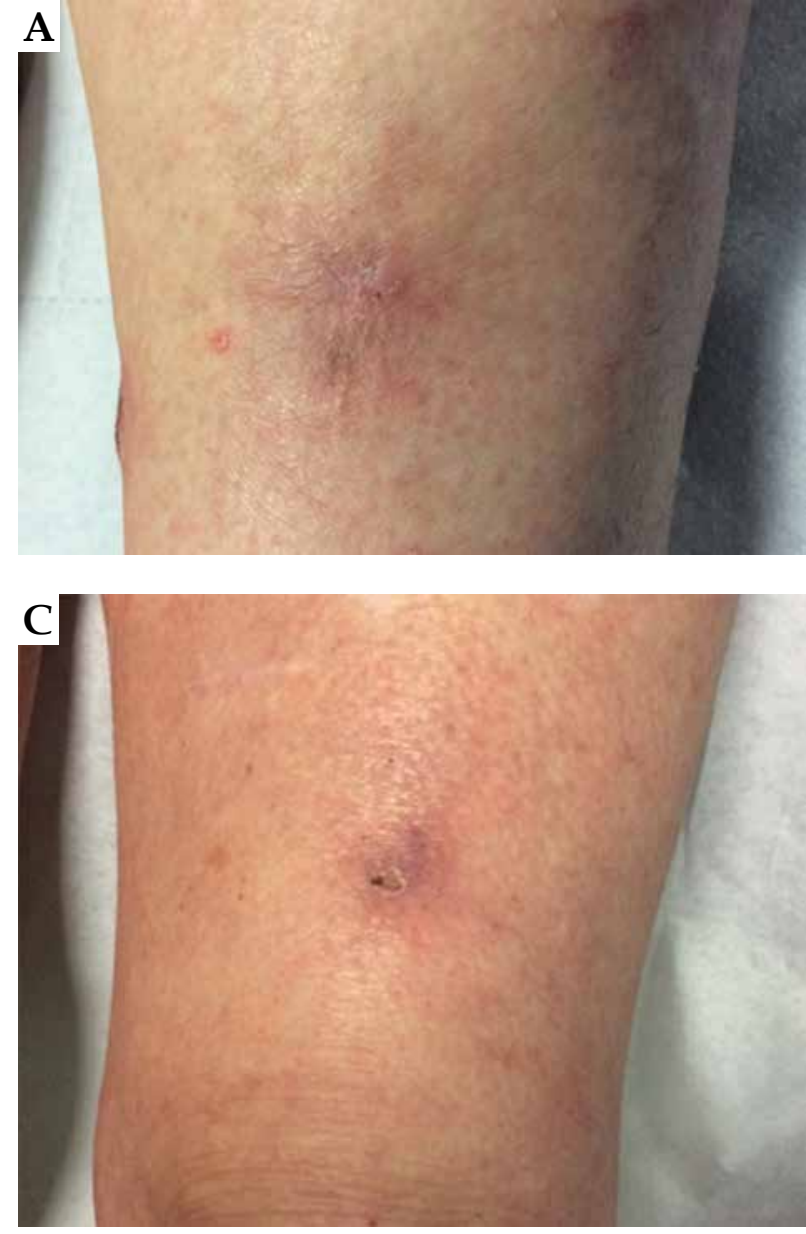

sible (previous irradiation, target difficult to reach, close proximity of organs at risk, multi-lesion diffused diseases, patient not able to sustain conventional fractionation), a partial radioablation (debulking) with DaRT would be able to trigger the awakening of the immune system. The immune system would then attack both the primary mass and any other lesion at further distance. This systemic anti-tumor immunity can be further augmented using immunoadjuvants and inhibition of immunosuppressive cells [34].

Another possible interpretation of our observations is that there is a possibility of misdiagnosis of KA as CSCC. The KA is a cryptic tumor, described for the first time in 1888, also named as 'pseudotumor', 'regressing tumor', or 'self-healing $\mathrm{SCC}^{\prime}$. As evidenced from other names used to indicate this distinct nosological entity, KA in a considerable percentage of cases may spontaneously regress, but rarely evolves into an invasive form of SCC $[35,36]$. For this reason, it stands in a gray area between malignancy and benignity, and many reports describe it as a subtype of SCC with low-grade malignancy. In contrast, some physicians believe that KA is essentially a benign lesion that can undergo malignant transformation into conventional SCC $[37,38,39]$. It is assumed to originate from the hair follicle and its triphasic nature (proliferative, stabilization, regression) seems to really mimic the phases of the cycle of hair follicle. KA shares some

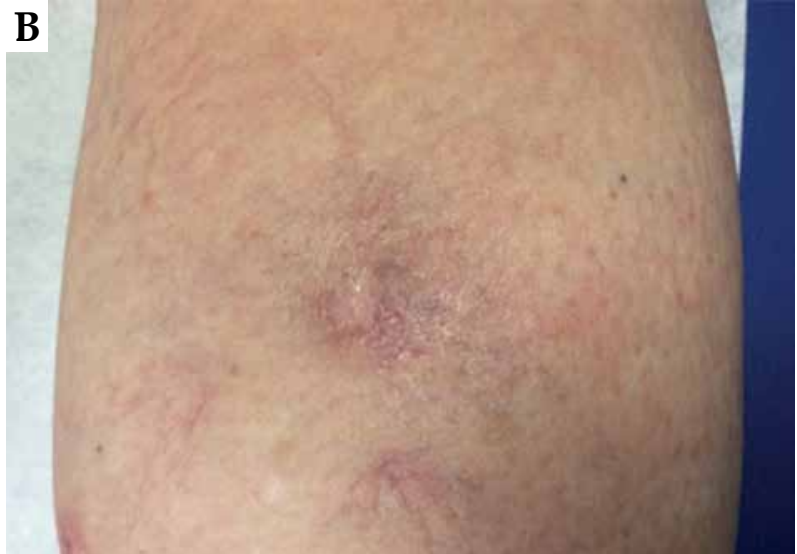

Fig. 8. Appearance of lesion A, B, and C at day 76 after implant

features with SCC, with similar cellular characteristics. A multitude of studies reveal no substantial differences between them not only clinically but also histopathologically.

In our study, the regression of untreated lesions $B$ and $C$ can be associated with an abscopal effect (AE) also because their regression time mismatch with the one observed for KA, which is between 3 to 6 months from the complete growth of lesion [40]. To our knowledge, this is the first case of an AE in a patient with multiple synchronous CSCC as well as the first case of an AE in a patient receiving DaRT treatment. We were able to observe this rare phenomenon in our patient also thanks to this unusual presentation of multiple synchronous lesions. By definition, we would not have had any chances to notice an AE if we had treated a patient with a solitary lesion.

\section{Conclusions}

In this study, according to our experience, we reported the first case of AE in CSCC. KA is very rare in multiple synchronous form and it gradually loses the capacity of spontaneous regression when it begins to exhibit characteristics of SCC. Furthermore, the occurrence time of KA spontaneous regression mismatches with the one observed in our patient, which was too fast, about 1 month against 3 to 6 months [40]. All these evidences 
support our hypothesis that an abscopal effect is stimulated by radiation and probably mediated by the immune system. In the next DaRT treatments, our intent is to monitor circulating soluble markers and immune cells such as T-lymphocytes. Largest changes from baseline in peripheral blood at different time points or induced de novo subpopulations can demonstrate indirectly the activation of the immune system mediated by radiation also in patients with solitary lesions, in which by definition, an abscopal effect cannot be observed. These evidences will further support recent findings on radiation used as immunological adjuvant in numerous clinical scenarios, also in advanced stage diseases [41,42]. Finally, DaRT technique is not complex; however, is still relatively new and requires standardization and specific training as other brachytherapy technique $[43,44,45,46]$.

\section{Ethics approval and consent to participate}

The patient is enrolled in the international 'first in man' clinical trial N.CTP-SCC-00 (NCT03015883); the protocol was approved by our regional ethical committee.

\section{Consent for publication}

The patient had signed an informed consent to publish information included in this case report.

\section{Availability of data and material}

All data generated or analyzed during this study are included in this published article and its supplementary information files.

\section{Funding} LTD.

This study was funded by the Alpha Tau Medical

\section{Disclosure}

Salvatore Roberto Bellia, Giacomo Feliciani, Massimo Del Duca, Manuela Monti, Valentina Turri, Anna Sarnelli, Ignazio Stanganelli, Giovanni Paganelli, Antonino Romeo, Toni Ibrahim report no conflict of interest.

Itzhak Kelson reports personal fees from the Alpha Tau Medical during conducting of the study; personal fees from the Alpha Tau Medical, outside the submitted work. In addition, Dr. Kelson has a patent for a radiotherapy device issued to the Alpha Tau Medical.

Yona Keisari reports grants and personal fees from the Alpha Tau Medical during the conduct of the study. In addition, Prof. Yona Keisari has a patent 'A radioactive surface source and a method for producing the same' pending.

Aharon Popovtzer reports grants and personal fees from the Alpha Tau Medical during the conduct of the study.

\section{References}

1. Mole RH. Whole body irradiation; radiobiology or medicine? Br J Radiol 1953; 26: 234-241.
2. Abuodeh $Y$, Venkat P, Kim S. Systematic review of case reports on the abscopal effect. Curr Probl Cancer 2016; 40: 25-37.

3. Rees GJ, Ross CM. Abscopal regression following radiotherapy for adenocarcinoma. Br J Radiol 1983; 56: 63-66.

4. Siva S, Callahan J, MacManus MP et al. Abscopal effects after conventional and stereotactic lung irradiation of non-smallcell lung cancer. J Thorac Oncol 2013; 8: e71-72.

5. Cotter S, Dunn GP, Collins KM et al. Abscopal effect in a patient with metastatic merkel cell carcinoma following radiation therapy: Potential role of induced antitumor immunity. Arch Dermatol 2011; 147: 870-872.

6. Maverakis E, Cornelius LA, Bowen GM et al. Metastatic melanoma - a review of current and future treatment options. Acta Derm Venereol 2015; 95: 516-524.

7. Stamell EF, Wolchok JD, Gnjatic $S$ et al. The abscopal effect associated with a systemic anti-melanoma immune response. Radiat Oncol Biol 2013; 85: 293-295.

8. Bramhall RJ, Mahady K, Peach AHS. Spontaneous regression of metastatic melanoma - clinical evidence of the abscopal effect. Eur J Surg Oncol 2014; 40: 34-41.

9. Barker CA, Postow MA. Combinations of radiation therapy and immunotherapy for melanoma: a review of clinical outcomes. Radiat Oncol Biol 2014; 88: 986-997.

10. Espenel S, Vallard A, Rancoule C et al. Critical reviews in oncology/hematology melanoma: last call for radiotherapy. Crit Rev Oncol Hematol 2017; 110: 13-19.

11. Ebner DK, Tinganelli W, Helm A et al. The immunoregulatory potential of particle radiation in cancer therapy. Front Immunol 2017; 8: 1-8.

12. Fernandez-Gonzalo R, Baatout S, Moreels M. Impact of particle irradiation on the immune system: From the clinic to mars. Front Immunol 2017; 8: 177.

13. Guinot JL, Rembielak A, Perez-Calatayud J et al. GECESTRO ACROP recommendations in skin brachytherapy. Radiother Oncol 2018; 126: 377-385.

14. Tagliaferri L, Kovács G, Aristei C et al. Current state of interventional radiotherapy (brachytherapy) education in Italy: results of the INTERACTS survey. J Contemp Brachytherapy 2019; 11: 48-53.

15. Kovács G, Tagliaferri L, Valentini V. Is an Interventional Oncology Center an advantage in the service of cancer patients or in the education? The Gemelli Hospital and INTERACTS experience. J Contemp Brachytherapy 2017; 9: 497-498.

16. Lancellotta V, Kovács G, Tagliaferri L et al. The role of personalized Interventional Radiotherapy (brachytherapy) in the management of older patients with non-melanoma skin cancer. J Geriatr Oncol 2019; 10: 514-517.

17. Lancellotta V, Kovács G, Tagliaferri L et al. Age is not a limiting factor in interventional radiotherapy (brachytherapy) for patients with localized cancer. Biomed Res Int 2018; 2018: 2178469.

18. Popovtzer A. Alpha radiation emitters device for the treatment of squamous cell carcinoma. https://clinicaltrials.gov/ ct2/show/NCT03015883.

19. Khan L, Choo R, Breen D et al. Recommendations for CTV margins in radiotherapy planning for non melanoma skin cancer. Radiother Oncol 2012; 104: 263-266.

20. Arazi L, Cooks T, Schmidt M et al. The treatment of solid tumors by alpha emitters released from 224 Ra-loaded sources - internal dosimetry analysis. Phys Med Biol 2010; 55: 12031218.

21. Cooks T, Arazi L, Schmidt M et al. Growth retardation and destruction of experimental squamous cell carcinoma by interstitial radioactive wires releasing diffusing alpha-emitting atoms. Int J Cancer 2008; 122: 1657-1664.

22. Cooks T, Tal M, Raab S et al. Intratumoral 224 Ra-loaded wires spread alpha-emitters inside solid human tumors in 
athymic mice achieving tumor control. Anticancer Res 2012; 32: 5315-5321.

23. Girdhani S, Lamont C, Hahnfeldt P et al. Proton irradiation suppresses angiogenic genes and impairs cell invasion and tumor growth. Radiat Res 2012; 178: 33-45.

24. Held KD, Kawamura H, Kaminuma T et al. Effects of charged particles on human tumor cells. Front Oncol 2016; 6: 23.

25. Hendry JH, Moore JV. The radiobiology of human cancer radiotherapy. Br J Cancer 1979; 40: 175

26. Antoniades J, Brady LW, Lightfoot DA. Lymphangiographic demonstration of the abscopal effect in patients with malignant lymphomas. Int J Radiat Oncol Biol Phys 1977; 2: 141-147.

27. Rees GJ. Abscopal regression in lymphoma: a mechanism in common with total body irradiation? Clin Radiol 1981; 32: 475-480.

28. Ohba K, Omagari K, Nakamura T et al. Abscopal regression of hepatocellular carcinoma after radiotherapy for bone metastasis. Gut 1998; 43: 575-577.

29. Uchida A, Mizutani Y, Nagamuta M et al. Effects of X-ray irradiation on natural killer (NK) cell system. I. Elevation of sensitivity of tumor cells and lytic function of NK cells. Immunopharmacol Immunotoxicol 1989; 11: 507-519.

30. Demaria $S, N g$ B, Devitt $M$ et al. Ionizing radiation inihibition of distant untreated tumors (abscopal effect) is immune mediated. Int J Radiat Oncol Biol Phys 2004; 58: 862-870.

31. Keisari Y. Tumor abolition and antitumor immunostimulation by physico-chemical tumor ablation. Front Biosci 2017; 22: 310-347.

32. Perez CA, Fu A, Onishko $\mathrm{H}$ et al. Radiation induces an antitumour immune response to mouse melanoma. Int J Radiat Biol 2009; 85: 1126-1136.

33. Gameiro SR, Jammeh ML, Wattenberg MM et al. Radiation-induced immunogenic modulation of tumor enhances antigen processing and calreticulin exposure, resulting in enhanced T-cell killing. Oncotarget 2014; 5: 403-416.

34. Confino H, Schmidt M, Hochman I et al. Inhibition of mouse breast adenocarcinoma growth by ablation with intratumoral alpha-irradiation combined with inhibitors of immunosuppression and CpG. Cancer Immunol Immunother 2016; 65: 1149.

35. Sanchez Yus E, Simon P, Requena L et al. Solitary keratoacanthoma: a self-healing proliferation that frequently becomes malignant. Am J Dermatopathol 2000; 22: 305-310.

36. Beham A, Regauer S, Soyer HP et al. Keratoacanthoma: a clinically distinct variant of well differentiated squamous cell carcinoma. Adv Anat Pathol 1998; 5: 269-280.

37. Hodak E, Jones RE, Ackerman AB. Solitary keratoacanthoma is a squamous-cell carcinoma: three examples with metastases. Am J Dermatopathol 1993; 15: 332-342.

38. Mandrell JC, Santa Cruz D. Keratoacanthoma: hyperplasia, benign neoplasm, or a type of squamous cell carcinoma? Semin Diagn Pathol 2009; 26: 150-163.

39. Weedon DD, Malo J, Brooks D et al. Squamous cell carcinoma arising in keratoacanthoma: a neglected phenomenon in the elderly. Am J Dermatopathol 2010; 32: 423-426.

40. Cassarino DS, De Rienzo DP, Barr RJ. Cutaneous squamous cell carcinoma: a comprehensive clinicopathologic classification. J Cutan Pathol 2006; 33: 261-279.

41. Demaria S, Formenti SC. Radiation as an immunological adjuvant: current evidence on dose and fractionation. Front Oncol 2012; 2: 153

42. Heguy A, Demaria S, Cooper B et al. Radiotherapy induces responses of lung cancer to CTLA-4 blockade. Nat Med 2018; 24: 1845-1851.

43. Autorino R, Vicenzi L, Tagliaferri L et al. A national survey of AIRO (Italian Association of Radiation Oncology) brachytherapy (Interventional Radiotherapy) study group J Contemp Brachytherapy 2018; 10: 254-259.
44. Tagliaferri L, Kovács G, Autorino R et al. ENT COBRA (Consortium for Brachytherapy Data Analysis): Interdisciplinary standardized data collection system for head and neck patients treated with interventional radiotherapy (brachytherapy). J Contemp Brachytherapy 2016; 8: 336-343.

45. Tagliaferri L, Budrukkar A, Lenkowicz J et al. Ent cobra ontology: the covariates classification system proposed by the Head \& Neck and Skin GEC-ESTRO Working Group for interdisciplinary standardized data collection in head and neck patient cohorts treated with interventional radiotherapy brachytherapy. J Contemp Brachytherapy 2018; 10: 260-266.

46. Tagliaferri L, Pagliara MM, Boldrini L et al. INTERACTS (INTErventional Radiotherapy ACtive Teaching School) guidelines for quality assurance in choroidal melanoma interventional radiotherapy (brachytherapy) procedures. J Contemp Brachytherapy 2017; 9: 287-295. 